\title{
The Principle and Applications of Business Promotion of E-commerce Enterprises Based on Big Data
}

\author{
Ke Liu, Jianhua Zhang, Fangfang Li \\ Institute of Management Engineering, Zhengzhou University, Zhengzhou, China \\ 610664336@qq.com
}

Keywords: Big Data, E-commerce Enterprises, Business Promotion

\begin{abstract}
The application value and promoting role of big data have been gradually affirmed with its application in various industry fields. And the deep application of big data by e-commerce companies based on online transactions is imperative. In this paper, the current state of business promotion of e-commerce companies is demonstrated through the classification and analysis of current various business promotion methods. Then it discusses the necessity of e-commerce enterprises to use big data for business promotion from the aspects of market demand and technology development. And then, through data collection and research analysis, the paper concludes the basic ideas of business promotion of e-commerce enterprises based on big data and the point of convergence between big data and e-commerce business promotion. In the end, the paper analyzes three challenges faced by e-commerce companies in business promotion and some suggestions were put forward.
\end{abstract}

\section{Introduction}

The development of big data is accelerating change from theoretical research into the application era since the "Big Data Year" (2013). E-commerce companies have natural advantages over many traditional industries because the behaviors of enterprises and consumers are all recorded in the form of data in real time. Some e-commerce companies have already recognized the huge potential value of big data and began building their own big data platform to improve some aspects of management and operations in order to increase sales. Nowadays, e-commerce services are developing constantly in a personalized way with the upgrade of consumer demand (Zhichao Liu, Yong Chen, Zhili Yao, 2014). For example, Alibaba, JD.com, eBay and Amazon, which are leading companies in the field of e-commerce are all building their own personalized service system based on big data to enhance the user experience. With the development of social economy, the variety of products and services is increasing and the amount of information is greatly expanded, and what follows is the reduction of data value density. If consumers want to obtain satisfactory products or services, it will inevitably cost more. If enterprises can master consumers' characteristics, and personalize the promotion method for each consumer based on the user data, it will not only improve customer delivered value and enhance customer satisfaction (Weiping Wang, 2017), but also help companies increase the conversion rate of potential users and increase sale in turn. However, the traditional promotion strategies have no competence to achieve this goal, but the 
advent of the big data can make it possible. For e-commerce companies, the promotion work in the era of big data is facing new challenges and opportunities for development.

\section{The Present Situation of E-Commerce Business Promotion}

For e-commerce companies, website is the foundation of transaction. Based on this, the business promotion of e-commerce companies can be divided into intra-site promotion and external promotion. It can also be divided into online promotion and offline promotion based on the Internet. At present, traditional and innovative ways are merged in the promotion strategy of enterprises, and following is the analysis of the current situation of e-commerce enterprises' business promotion from three aspects: inside promotion, outside promotion and the application of big data in e-commerce promotion.

\subsection{The Intra-Site Promotion}

As an important part of promotion affairs, intra-site promotion affects the user experience and determines the retention of potential users to a large extent. The promotion in the website largely relies on the recommendation system to recommend some products or services to existing customers they may like or need. With the enrichment of products, the recommendation system should be able to recommend suitable product or service information to specific customer (Qian Wang, Li Qian, 2014) based on the data like user's real-time behavior, purchase records to optimize customer experience. And it requires e-commerce companies to develop this system alone to a large degree.

The most typical case is that Amazon collects, analyzes, and understands customer behavior data to personalize recommendation service and provide thoughtful services to different customers. This not only identifies the moment when the user generates the purchase idea, but also increases the production rate of the user's effective purchase behavior. At the same time, it shortens the purchase path and time of users, optimizes the user experience, and reduces the unwarranted harassment of the traditional promotion method to the customer. It is a win-win process for users and enterprises. At present, most e-commerce companies have their own recommendation systems, but the actual application results are uneven, and there are not many that can achieve Amazon's level.

\subsection{External Promotion}

External promotion is largely to promote website through the promotion of products and services to potential customers. And it can also be divided into online and offline parts. Among them, online promotion belongs to network promotion. At present, the network promotion methods that e-commerce enterprises apply are broadly divided into the following categories.

(1)Search engine promotion. As the name suggests, it is to use search engine to implement the promotion work and mainly includes the following ways:

- Search engine registration. It's the most basic promotion method that every e-commerce company should adopt.

- Search engine optimization (SEO). It is to make the website more suitable for the search engine index rules and keep it in the forefront of search results as much as possible. SEO is not put things right once and for all but a continuous process. The application of SEO is very extensive.

- Keyword advertising. It has certain pertinence and is a cost-effective way to promote the website, but the relevant keywords is to much that enterprises should find out the most valuable words to achieve ideal effects. 
Search engine promotion, having a certain degree of precision can push relevant advertisements to users based on their search content, but it is based on that the user clearly know what he/she needs, and there are still defects in early insight into user intent.

(2) Email promotion based on the user license. It is an old-fashioned network promotion method which helps to reduce the user's dislike and enhance the corporate image. The e-mails which have appropriate content and reasonable period always have a good promotion effect.

(3) Promotion of resources cooperation. This allows users to discover their own websites from the cooperative website, and achieve the purpose of mutual promotion (Azhen Ye, 2014). The key of this promotion method is to explore the resource advantages and needs of itself and the other party, so as to achieve a win-win effect.

(4) Promotion of online advertising. There are several types of online advertisements like web advertisements, software bundle advertisements, etc.; With the development of online media, new forms of advertising are constantly emerging on the Internet. For example, information flow advertisements which can integrate well with users' daily entertainment activities, which has a good development prospects. Not limited to the above, a variety of network promotion methods can be understood as different forms of online advertising.

(5)Social media promotion. Tthe company uses some social media platforms such as Weibo and WeChat to publish related products and services, and uses the fans' attention and community effects, such as Weibo accounts and WeChat public accounts which have a large amount of fans to increase the exposure of companies on social networks and increase the brand awareness in the end (Hong $\mathrm{Lu}, 2014)$. The user groups already classified make this type of promotion achieve the goal effectively.

Offline promotion is an important promotion part that e-commerce companies can't ignore. With the concept of "new retail” is proposed, people doesn't over-emphasize the difference between online and offline frequently, and omni-channel marketing becomes the future development trend. According to iResearch "Annual Data Release - E-Commerce 2017", the transaction volume of China's online shopping market in 2017 increased by 29.6\% compared with last year, reaching 6.1 trillion yuan. At the same time, iResearch believes that this is because 2017 is the first practice year that online and offline market begin to integrate together. It have begun to work that the offline work can guide customers to online store and the online market can empowerment the data from the offline, which has injected new vitality into online market. At present, offline promotion should be promoted together with online promotion, so that consumers can receive consistent promotion information from various channels and deepen their impression. The collection and analysis of consumers' offline behavioral data, and the transformation of traditional promotion thinking is also an important task for e-commerce companies.

\subsection{The Application of Big Data in Promotion}

With the development of big data and other information technologies and new Internet media, people start to innovate some traditional promotion methods, and some new promotion methods have been derived. The typical ones are as follows:

- Search engine precision marketing. Search engine vendors describe the user's basic attributes, long-term preferences and short-term behaviors by integrating and analyzing the data of the user's search behavior, and accordingly present accurate advertising content on the advertising alliance website for the audience with above characteristics.

- Real Time Bidding.The entire chain includes four parts: Demand-Side Platform(DSP), Data Management Platform(DMP), Ad Exchange and Sell-Side Platform(SSP) for Internet media. The advertiser puts the advertising demand on the DSP, and the Internet media puts its own advertising 
resources on the advertising trading platform. The DSP completes the bidding purchase through the DMP's analysis on the ad exchange platform. The implementation effect is that when a user browses a certain product or clicks on a certain type of advertisement, and the browsing behavior will be recorded in the cookie. Through the advertisement trading platform, the user will be recommended with the relevant advertisement when browsing the webpage next time. This kind of promotion method greatly improves the accuracy of advertising.

- Redirected Precision Marketing. It is also known as remarketing which is about how to help companies generate repeat customers. Redirected Precision Marketing try to get consumers who have visited the website but don't have purchase behavior to visit the website again or make them actually purchase the products or services. It make efforts to improve the conversion rate and recover customers who may have lost.

The effect of new promotion methods based on the big data is remarkable.

\section{The Necessity of E-Commerce Enterprises to Use Big Data for Business Promotion}

Through the above summary and analysis of the existing promotion methods, the emerging promotion method based on big data can obviously improve the conversion rate of potential users and various traditional network promotion methods are also gradually using big data to adjust and optimize, so that marketing information can reach potential target users accurately. The following is the analysis of the necessity of e-commerce companies to implement business promotion based on big data from the aspects of the upgrade of market demand and the development of technology.

\subsection{The Upgrade of Market Demand}

With the development of the social economy, people's living standards have greatly improved, and the concept of consumption has also undergone dramatic changes (Linan Ruan, 2016). In today's highly developed Internet and prosperous market, on the one hand, information asymmetry is greatly reduced, consumers can easily understand the information of many options, and on the other hand, the variety of goods is increasing, which also makes it difficult to pick out target information. . In addition, most consumers are more concerned about the consumer experience and whether the goods can meet their individual needs, which puts new demands on the promotion work. E-commerce companies should find potential consumers according to their own product characteristics, or in other words, according to the mastery of consumer preference to predict the needs of potential consumers accurately, bringing a good experience and making a good impression for customers from the beginning.

Based on big data, it can not only help predict the potential needs of users, but also grasp consumer preferences through user characteristics, thus satisfying consumers' individual needs.

\subsection{The Development of Technology}

In the era of big data, with the explosive growth of data, big data technology is also in constant progress and becoming more and more popular in various industries. In addition, the e-commerce industry faces fierce market competition and how to stand out among many e-commerce companies and avoid homogenization is an urgent problem for enterprises to solve, and the combination of e-commerce promotion and big data is an opportunity. At present, small and medium-sized e-commerce companies rely more on big data platforms to collect and analyze customer behavior data, so as to obtain user preferences and tap potential customers. Although many small and medium-sized e-commerce companies do not have the advantage of big data technology, some large enterprises, such as Alibaba, JD, Baidu, Tencent, etc., have developed many data products, and 
based on this data expansion, they sold these products as data services to small and medium-sized e-commerce companies. And a new commercial ecosystem has been formed (Yang Zheng, $\mathrm{Li} \mathrm{Yu,}$ 2016). Therefore, whether it is a large or small-scale e-commerce enterprise, there are ways to use the big data to achieve accurate promotion.

\section{Improved Method for E-Commerce Promotion Based On Big Data}

With the upgrading of consumer demand, mastering the personalized characteristics of consumers has become one of the goals of big data analysis, and the data mining technology located in the important position of the big data technology framework is the key to achieve this goal. It is also a key technology for e-commerce companies to promote their business based on big data. For e-commerce companies, how to transform, construct and implement business promotion ideas based on big data in the era of big data is the key to improve the efficiency of promotion. Cognition is the premise of application. Only by understanding the combination point of big data and e-commerce business promotion can data mining and e-commerce promotion be effectively combined to play the value of big data. The following sections will present the basic ideas of e-commerce enterprise business promotion based on the integration of the general process of promotion and process of big data mining.

\subsection{The Basic Idea of Business Promotion Based on Big Data}

Data mining is a iterative process of continuous optimization (Liping Yan,2016), and its content and sequence are roughly shown in Figure 1.

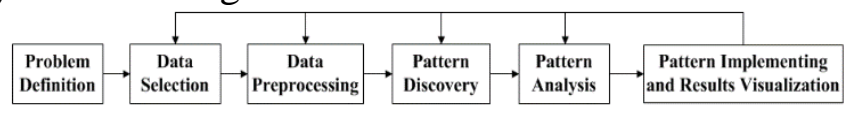

Figure 1 Data mining process.

Synthesizing the process of big data mining and the general process of promotion, the basic idea of business promotion based on big data is also an iterative process of continuous optimization. Data analysis runs through the idea, which can be summarized as four steps as shown in Figure 2: data collection, target selection, promotion strategy formulation, effect estimation, promotion strategy implementation, and effect monitoring.

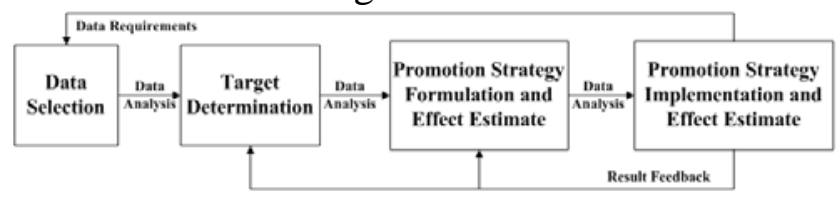

Figure 2 The basic idea of business promotion based on big data.

\subsubsection{Market Research}

Market research is a basic work that should be conducted in any marketing work. The arrival of the era of big data can help companies break through the shortage of sample size, representativeness and timeliness, making the survey results more representative and timely. In the era of big data, almost all information and behaviors of online consumers can be recorded in the form of data, and the market is composed of consumers. E-commerce companies should collect relevant data that is conducive to the implementation of the promotion according to its own positioning. So that market research based on the big data can help e-commerce companies to understand the market situation in depth. At the same time, while collecting data, it will certainly generate a large amount of new data along with the user's new behavior. Enterprises should update the data in incremental update 
mode to improve data collection efficiency (Xiangwu Meng, Weiyu Ji, Yujie Zhang, 2015). The information that enterprises need to collect generally includes consumer attribute data, behavior data, and so on. And the sources of these data can be roughly divided into the following three categories.

- First party data source. Internal data accumulated by the daily operation of the enterprise, including product data, sales data, customer relationship management data, etc., which can reflect the characteristics of the product and the target customers, and the enterprise can find out some specific characteristics of potential customers based on the existing customers. Though this kind of data source is high quality, it still lacks breadth and depth.

-Second-party data source. It's the data that comes from partners. Many companies choose to cooperate with each other to share data resources. For example, in the cooperation between JD.com and Tencent, JD.com combines its own e-commerce big data with Tencent's social data to master the user's accurate portraits, and thus achieve accurate promotion. Tencent can also understand the user's consumption habits through JD's e-commerce platform. So it is able to recommend ads or other content to users that match their characteristics and preferences better.

- Third-party data source. It is the data that comes from data providers such as professional research companies. This kind of data can help enterprises to expand the breadth and depth of data to a large extent, so that enterprises can grasp the image of consumers more deeply.

\subsubsection{Target Selection}

Most of the traditional promotion work just divides the market according to the shallower level such as age and geographical location and select the target customer from them. This kind of division is so rough that the promotion work cannot be refined. But the arrival of the era of big data allows enterprises to differentiate users from more and more detailed dimensions through data mining technology, master their differentiated features, and then select target customer groups based on their market positioning. Enterprises can still further divide the selected target groups, so that the promotion has the basis to achieve differentiation and personalization (Xiangming Wei, Jing Zhang, Xiansong Xiang, 2016). After having a large number of customers, enterprises can use the clustering algorithm of big data mining to classify the existing customers into more and more detailed dimensions, and then classify unknown customers by classification algorithm to identify potential customers.

In summary, big data helps e-commerce companies to divide users more precisely, so they can accurately identify and select promotion targets, make promotion goals clear, and improve promotion efficiency in the end.

\subsubsection{Promotion Strategy Formulation and Effect Estimation}

After selecting the promotion target, the next step is to formulate a specific promotion strategy to attract target potential customers. The enterprise can analyze the consumer's attribute data, social data, behavior data, etc., and grasp the behavior characteristics of the consumer's personality and preference, using association rules or decision tree models to predict their needs. Companies should choose the promotion methods and channels that best attract the target audience, and push the information of the products or services that they are most likely to be interested in at the right time. After preliminarily determining the promotion strategy, enterprises can also use the neural network algorithm with the advantages of self-adaptation, self-learning and high fault tolerance to predict the promotion effect by grasping the law of website traffic, so that the distribution of promotion investment is more reasonable (Xueqiang Luo, Bin He, Xiangtian Xie, 2012). It provide a certain reference for the revision and formulation of corporate promotion strategies. 


\subsubsection{Promotion Strategy Implementation and Effect Monitoring}

After the promotion strategy is finalized, the next step is implementation, as a system of work, people should monitor the implementation process and evaluate the promotion effect continuously, analyze whether the pushed goods or service information is accepted. According to the evaluation, the promotion staff can adjust and optimize each promotion method and content continuously, so that the comprehensive promotion effect of various promotion methods can be optimized, and it can also help enterprises to recover lost customers. Big data provides a means for multi-faceted and real-time evaluation of promotion effects.

\section{The challenge of e-commerce promotion based on big data}

\subsection{E-Commerce Enterprises are Seriously Homogenized}

With the continuous expansion of the development scale of e-commerce, the homogenization competition between e-commerce enterprises is more and more serious (Haijian Gao, 2015). The business model of enterprises like Tmall, Jingdong Mall, Amazon China, Dangdang, is nothing but a platform plus self-operation. The bargaining power of the merchants who are invited to enter the platform has increased. At the same time, this has further caused the homogenization of the products of e-commerce platforms. If the price is different, how to make up for the price disadvantage, and if the price is the same, how to obtain the user preference are questions that the e-commerce platforms need to think about. Due to the increase in consumer choices, the homogenization of e-commerce companies has made it difficult to promote their work. How to stand out among many e-commerce companies without homogenization is the key that the e-commerce enterprises maintain its vitality and achieve sustainable profitability. In the era of big data, e-commerce companies should use big data to inspire innovative thinking, develop unique product and service models to form their own competitive advantages, make the promotion have a material basis, and thus improve the promotion efficiency.

\subsection{Technical Restrictions}

Although the big data environment has been initially formed in China, the big data processing and analysis technology, which is the core element of performing the value of big data, is still mastered by some large enterprises. Although the thriving development of third-party data service companies provides a means for small and medium-sized enterprises to utilize big data, the degree of fitting with self condition is still not satisfactory. At the same time, the market has not yet formed a unified industry standard and the data islands between enterprises still exist. Different enterprises understand and define data from their respective perspectives, which increases the communication cost and greatly reduces the utilization of data. In the end, it affects the efficiency of promotion in turn.

E-commerce companies should recognize the importance of data construction and development, gradually internalize the services provided by third-party data service companies, and gradually establish and utilize their own data assets. In addition, leading companies in some industries should exert their influence, improve the unified big data technology and operational standards, establish a big data sharing mechanism, and maximize the comprehensive value of data (Abdulzheman Abu Dhabi, Nirupar Aishan, 2017). 


\subsection{User’s Privacy Protection Issues}

As a scientific technology, big data is a double-edged sword with advantages and disadvantages. E-commerce companies may disclose user information while promoting based on user data, especially in the era of big data when most of the user's behavior will be digitized. E-commerce companies collect various data of users, which in turn will cause users to be disgusted and worried about their privacy leaks. In recent years, news about user privacy leaks has emerged, and people's concerns about data security are also increasing. . Since enterprises are the beneficiaries of data, they naturally have to be responsible for data security, and at the same time avoid over-expansion of data collection.

\section{Conclusions}

This paper mainly classifies the current various business promotion methods and systematically summarizes the current status of business promotion of e-commerce enterprises. And it discussed the necessity of using bid data to implement promotion work from the aspects of the promotion problems, market demand and technology development. Next, combined with the implementation process of big data mining and its integration with e-commerce business promotion, the basic ideas of business promotion based on big data are summarized. In the end, this paper analyzes three challenges faced by e-commerce companies in business promotion and some suggestions were put forward. Hope it can provide ideas for e-commerce companies to explore how to effectively use big data and related technologies to optimize existing promotion methods.

\section{References}

[1] Zhichao Liu, Yong Chen, Zhili Yao, 2014. Innovation of E-commerce Service Model in the Age of Big Data, Science and Technology Management Research. 34(01), pp.31-34.

[2] Weiping Wang, 2017. Research on E-commerce Precision Marketing Strategy Based on Big Data Environment, Modern Business. (30), pp.26-27.

[3] Qian Wang, Li Qian, 2014. Analysis on the Development Trend of E-Commerce Personalized Referral Service in Big Data Environment, Commercial Research. (08), pp.150-154.

[4] Azhen Ye, 2014. Research on optimizing the promotion strategy of e-commerce website for small and medium sized enterprises, E-Business Journal. (11), pp.46-47.

[5] Hong Lu, 2014. The external promotion strategy of small-scale foreign trade e-commerce platform, Enterprise Econom. 33(12), pp.39-43.

[6] Linan Ruan, 2016. Application Research of Precision Marketing in Jingdong in the Big Data Era, University of Electronic Science and Technology of China.

[7] Yang Zheng, Li Yu, 2016. Analysis of PESTEL Model for E-commerce Development in Big Data Environment, Rural Economy and Science-Technology. 27(08), pp.131-132.

[8] Liping Yan,2016. Application of Web Data Mining Technology in E-commerce, Computer Era. (03), pp.43-44+49.

[9] Xiangwu Meng, Weiyu Ji, Yujie Zhang, 2015. Recommendation system in big data environment, Journal of Beijing University of Posts and Telecommunications. 38(02), pp.1-15.

[10] Xiangming Wei, Jing Zhang, Xiansong Xiang, 2016. Precision $\quad$ Marketing Based on Big Data, Enterprise Management. (11), pp.91-93.

[11] Xueqiang Luo, Bin He, Xiangtian Xie, 2012. Promotion decision of e-commerce website based on Neural Network, Enterprise Economy. 31(11), pp.87-90.

[12] Haijian Gao, 2015. Research on E-commerce Industry Based on Big Data Perspective, Capital University of Economics and Business.

[13] Abdulzheman Abu Dhabi, Nirupar Aishan, 2017. E-commerce precision marketing strategy from the perspective of big data, China Statistics. (10), pp.23-25. 